\title{
Bulk one-point function on disk in one-matrix model
}

\author{
Alexander Belavin 1 and Chaiho Rim 2 \\ ${ }^{1}$ L.D. Landau Institute for Theoretical Physics, Chernogolovka 142432, Russia \\ ${ }^{2}$ Dept. of Physics and Center for Quantum Spacetime, Sogang University, Seoul 121-742, Korea
}

\begin{abstract}
We consider bulk correlation numbers on disk in one-matrix model. Using the recently found so-called resonance transformation from the $\mathrm{KdV}$ to the Liouville frame 17, we obtain an explicit expression for the bulk one-point function. The result is consistent with the form of FZZ one-point function[20] in the boundary Liouville Field Theory.
\end{abstract}

\section{Introduction}

Since the middle of 80's, there exist two independent approaches to 2D Quantum Gravity: the continuous one called Liouville Gravity (LG) [1, 2, 3, 4] and the discrete one using the techniques of Matrix Models (MM) [5, 6, 7, 8, 9, 10, 11. One may refer to more references in reviews in [12, 13].

Comparison of some quantities calculated in a number of particular models using both approaches confirms the expectation about their equivalence[11, 14, 15]. The most easily checked quantities in MM are the $n$-point "correlation numbers"

$$
C_{k_{1}, \cdots, k_{n}}=\left\langle O_{k_{1}} \cdots O_{k_{n}}\right\rangle
$$

where $O_{k}$ is the integrated form of the local density (2-form operator) $\mathcal{O}_{k}(X)$

$$
O_{k}=\int_{\mathcal{M}} \mathcal{O}_{k}(X)
$$

over the manifold $\mathcal{M}$ which accommodates both "matter" and the metric degrees of freedom localized at $X \in \mathcal{M}$.

\footnotetext{
${ }^{1}$ e-mails: belavin@itp.ac.ru

${ }^{2}$ e-mail: rimpine@sogang.ac.kr
} 
It is convenient to introduce a generating function of the correlation numbers in Liouville gravity

$$
Z_{L G}\left(\left\{\lambda_{k}\right\}\right)=\left\langle e^{\sum_{k} \lambda_{k} O_{k}}\right\rangle
$$

which can be viewed as the partition function of the original theory perturbed by adding the fields $\mathcal{O}_{k}(X)$ to the action density with the coupling constant $\lambda_{k}$. A similar partition function, which depends on the parameters $t_{k}$, can be introduced in Matrix models.

However, a naive identification of observables and their correlation numbers obtained from two approaches does not agree in general. The reason for this difficulty lies in the ambiguity of the choice of contact terms [16, 17]. This ambiguity is equivalent to the freedom in the identification of the coupling parameters $\lambda_{k}$ in LG with the ones $t_{k}$ in MM of the same gravitational dimensions.

It was conjectured in [16] that there exists a special choice of the contact terms in $\mathrm{MM}$ or, equivalently, the special transformation $t_{k}=t_{k}\left(\left\{\lambda_{j}\right\}\right)$, which ensures the coincidence of the partition functions in the Minimal Liouville gravity MLG $(2,2 \mathrm{p}+1)$ and in the $p$-critical one-matrix model $\mathrm{OMM}(\mathrm{p})$ for the random surfaces with the sphere topology. This relation between the parameters $t_{k}$ and $\lambda_{k}$ was obtained in [16] up to the linear terms. Its explicit form to all orders was conjectured in [17] and checked up to the 4 -th order [17, 18].

The existence of a similar transformation for the general $\left(p_{1}, p_{2}\right)$ case remains an open problem, and so much as the problem of the calculation of the correlation numbers and their comparison on the surface with non-spherical topology.

In this note, we consider the non-spherical topology, namely, the fluctuating disk and propose the explicit expression for the generating function of the $n$-point bulk correlation numbers on disk. In our calculation, we use the string equation [17] in the conformal frame.

Particularly, we compute the partition function and the one-point function for the one-matrix model in this topology, which were obtained earlier in [16] by means of a different way. We find our results consistent with the form of the Fateev-ZamolodchikovZamolodchikov one-point function [20] in the boundary Liouville Field Theory.

In section 2 we give a short summary of the known results on the $\operatorname{OMM}(\mathrm{p})$. In section 3, the partition function on the random disk with a fixed length of the boundary and one-point function of the bulk operator in the $\mathrm{OMM}(\mathrm{p})$ are calculated. In section 4. the corresponding answers are compared with the Liouville gravity results. Section 5 is the conclusion and discussion.

\section{One-Matrix Model}

It was shown in [5, 6, 7] that the scaling partition function of the $\operatorname{OMM}(\mathrm{p})$ is expressed in terms of the susceptibility $u_{*}\left(t_{k}\right)$ which corresponds to the suitably chosen root of 
the string equation $P\left(u_{*}\right)=0$. Here, $P(u)$ is the $p+1$-degree polynomial of $u$

$$
P\left(u, \mu,\left\{t_{k}\right\}\right)=u^{p+1}-\mu u^{p-1}+\sum_{k=1}^{p-1} t_{k} u^{p-k-1}
$$

where $\mu$ is the cosmological constant, and the parameters $t_{k}$ describe the relevant deviations from the $p$-critical point.

The singular (or universal) part of the partition function is expressed[17] as

$$
Z\left(\mu,\left\{t_{k}\right\}\right)=\frac{1}{2} \int_{0}^{u_{*}} d u P^{2}\left(u, \mu,\left\{t_{k}\right\}\right) .
$$

It was conjectured and partly checked in [16, 17] that there exists an analytic transformation $t_{k}=t_{k}\left(\mu,\left\{\lambda_{j}\right\}\right)$ compatible with the scale properties so that the correlation numbers, defined by expanding the partition function into series of the new variables $\lambda_{k}$, satisfy the same restrictions to be fulfilled in the minimal Liouville gravity. After this transformation the polynomial $P\left(u, t_{k}\right)$, as a function of the new parameters $\lambda_{k}$, takes the form (up to the factor $(p+1) ! /(2 p-1) ! !)$

$$
\mathcal{P}\left(u, \mu,\left\{\lambda_{k}\right\}\right) \equiv P\left(u, \mu,\left\{t_{k}(\lambda)\right\}\right)=u_{0}^{p+1} \mathcal{Q}\left(u / u_{0},\left\{\lambda_{k}\right\}\right)
$$

where

$$
\begin{aligned}
\mathcal{Q}\left(x,\left\{\lambda_{k}\right\}\right) & =\sum_{n=0}^{\infty} \sum_{k_{1} \ldots k_{n}=1}^{p-1} \frac{\lambda_{k_{1}} \lambda_{k_{2}} \cdots \lambda_{k_{n}}}{n !}\left(\frac{d}{d x}\right)^{n-1} L_{p-\sum k_{i}-n}(x), \\
\left(\frac{d}{d x}\right)^{-1} L_{p}(x) & \left.\equiv \int_{0}^{x} L_{(} y\right) d y=\frac{L_{p+1}(x)-L_{p+1}(x)}{2 p+1}
\end{aligned}
$$

$u_{*}(\lambda)$ is the suitably chosen root of $\mathcal{P}\left(u, \lambda_{k}\right)$ and reduces to $u_{0}$ if $\lambda_{k}=0$ as given in Eq. (3.26) of [17]

$$
u_{0}=\sqrt{\frac{2(2 p-1)}{p(p-1)} \mu} .
$$

The partition function in the new variables is rewritten as

$$
\mathcal{Z}\left(\mu,\left\{\lambda_{k}\right\}\right)=\frac{1}{2} \int_{0}^{u_{*}} \mathcal{Q}^{2}\left(u / u_{0}, \lambda_{k}\right) d u
$$

and is considered as the generating function of the correlation numbers in the Liouville frame.

It is important to remember that these new correlation numbers are equal to the coefficients of the expansion of the partition function $\mathcal{Z}\left(\mu,\left\{\lambda_{k}\right\}\right)$ around the point $\lambda_{k}=0$ which does not coincide with the point $t_{k}=0$. The last one was used to define the correlation numbers in the $\mathrm{KdV}$ frame. We will refer to the new choice of the point of the expansion as the Liouville (or the conformal) background following [16]. 


\section{Bulk one-point function on disk}

Now we consider the fluctuating disk with the boundary length $L$. It was shown in [7, 19] that the partition function of the random disk with a finite boundary is given by

$$
Z_{B}\left(\mu, L, t_{1}, \cdots, t_{p-1}\right)=\frac{1}{\sqrt{L}} \int_{t_{p-1}}^{\infty} d y e^{-L u(y)}
$$

where $u(y)$ stands for the solution of the string equation in the KdV frame

$$
P\left(u, \mu, t_{1}, \cdots, t_{p-2}, y\right)=u^{p+1}-\mu u^{p-1}+\sum_{k=1}^{p-2} t_{k} u^{p-k-1}+y=0 .
$$

The disk partition function $Z_{B}\left(\mu, L, t_{k}=0\right)$ in the $\mathrm{KdV}$ frame was computed by Moore, Seiberg, and Staudacher. The answer is given in Eqs. (4.4) and (4.5) of [16].

However, to compare the results of MM with the ones of MLG on disk we need to use the Liouville frame. Therefore, we will start from the formula

$$
\mathcal{Z}_{B}\left(\mu, L,\left\{\lambda_{k}\right\}\right)=\frac{1}{\sqrt{L}} \int_{\lambda_{p-1}}^{\infty} d y e^{-L u(y)}
$$

where $u(y)$ stands for the solution of the string equation in the Liouville frame

$$
\mathcal{P}\left(u, \mu, \lambda_{1}, \cdots, \lambda_{p-2}, y\right)=0 .
$$

We can compute the partition function and the $n$-point functions on disk using the string equation after changing the integration variable $y$ to $u$. For this we use the relation

$$
\frac{d y}{d u}=-\frac{d \mathcal{P}\left(u, \mu,\left\{\lambda_{k}\right\}\right)}{d u}
$$

and obtain

$$
\begin{aligned}
\mathcal{Z}_{B}\left(\mu, L,\left\{\lambda_{k}\right\}\right) & =-\frac{u_{0}^{p+1}}{\sqrt{L}} \int_{x_{*}}^{\infty} d x \frac{d Q\left(x,\left\{\lambda_{k}\right\}\right)}{d x} e^{-L u_{0} x} \\
& =u_{0}^{p+2} \sqrt{L} \int_{x_{*}}^{\infty} d x Q\left(x,\left\{\lambda_{k}\right\}\right) e^{-L u_{0} x}
\end{aligned}
$$

where the integration by parts is performed and $x_{*}$ is the corresponding root of the renormalized string equation $Q\left(x_{*},\left\{\lambda_{k}\right\}\right)=0$. The formula (3.6) is the expression for the generating function of the correlation numbers on the disk in the Liouvile frame. The disk partition function is obtained when $\lambda_{k}=0$

$$
\mathcal{Z}_{B}\left(\mu, L,\left\{\lambda_{k}\right\}=0\right)=\frac{u_{0}^{p+1}}{\sqrt{L}} \int_{1}^{\infty} d x L_{p}(x) e^{-L u_{0} x} .
$$


The bulk one-point function is given as

$$
\begin{aligned}
\left\langle O_{k}\right\rangle_{L} & =\left.\frac{\partial}{\partial \lambda_{k}} \mathcal{Z}_{B}\left(\mu, L, \lambda_{1}, \cdots, \lambda_{p-1}\right)\right|_{\left\{\lambda_{i}\right\}=0} \\
& =u_{0}^{p-k} \sqrt{L} \int_{x_{*}}^{\infty} d x L_{p-1-k}(x) e^{-L u_{0} x}
\end{aligned}
$$

One can evaluate the expressions using Eqs. (3.7) and (3.8), and the relation [17] between the Legendre polynomials and the Macdonald function of a half-integer order

$$
\int_{1}^{\infty} d x L_{n}(x) e^{-p x}=\sqrt{\frac{2}{\pi p}} K_{n+1 / 2}(p) .
$$

The explicit results are

$$
\begin{aligned}
\mathcal{Z}_{B}\left(\mu, L, \lambda_{k}=0\right) & =\sqrt{\frac{2}{\pi}} \frac{u_{0}^{p+1 / 2}}{L} K_{p+1 / 2}\left(u_{0} L\right) \\
\left\langle O_{k}\right\rangle_{L} & =\sqrt{\frac{2}{\pi}} u_{0}^{p-k-1 / 2} K_{p-k-1 / 2}\left(u_{0} L\right) .
\end{aligned}
$$

These relations coincide with formulas (4.19) and (4.24) in [16] where they were obtained in a different way.

\section{Comparison with Boundary Liouville field theory}

Noting that the Liouville gravity partition function with the fixed boundary length $\ell$, $Z_{B L G}\left(\mu, \ell,\left\{\lambda_{k}\right\}\right)$ is related to the one with the fixed boundary cosmological constant $\mu_{B}, \mathcal{Z}\left(\mu, \mu_{B},\left\{\lambda_{i}\right\}\right)$ by the inverse Laplace transform

$$
Z_{B L G}\left(\mu, \ell,\left\{\lambda_{k}\right\}\right)=\ell \int_{\uparrow} \frac{d \mu_{B}}{2 \pi i} e^{\mu_{B} \ell} Z_{B L G}\left(\mu, \mu_{B},\left\{\lambda_{k}\right\}\right),
$$

where the contour $\uparrow$ goes along the imaginary axis to the right from all the singularities of the integrand.

One can express the one-point correlation number with fixed $\ell$ in terms of the one with fixed $\mu_{B}$ as

$$
\left\langle O_{k}\right\rangle_{\ell}=\ell \int_{\uparrow} \frac{d \mu_{B}}{2 \pi i} e^{\mu_{B} \ell}\left\langle O_{k}\right\rangle_{\mu_{B}}
$$

where

$$
\left\langle O_{k}\right\rangle_{\mu_{B}}=\left.\frac{\partial}{\partial \lambda_{k}} Z_{B L G}\left(\mu, \mu_{B},\left\{\lambda_{i}\right\}\right)\right|_{\left\{\lambda_{i}\right\}=0} .
$$

The bulk one-point function in the Boundary Liouville field theory is obtained in 20.

$$
U\left(\alpha \mid \mu_{B}\right)=\frac{2}{b}\left(\pi \mu \gamma\left(b^{2}\right)\right)^{(Q-2 \alpha) /(2 b)} \Gamma\left(2 b \alpha-b^{2}\right) \Gamma\left(\frac{2 \alpha}{b}-\frac{1}{b^{2}}\right) \cosh ((2 \alpha-Q) \pi s)
$$


with

$$
\cosh ^{2}(\pi b s)=\frac{\mu_{B}^{2}}{\mu} \sin \left(\pi b^{2}\right)
$$

Using the Laplace transform, one gets

$$
\begin{aligned}
U\left(\alpha \mid \mu_{B}\right) & =\int_{0}^{\infty} \frac{d \ell}{\ell} e^{-\mu_{B} \ell} W_{\alpha}(\ell) \\
W_{\alpha}(\ell) & =\frac{2}{b}\left(\pi \mu \gamma\left(b^{2}\right)\right)^{(Q-2 \alpha) /(2 b)} \frac{\Gamma\left(2 b \alpha-b^{2}\right)}{\Gamma\left(1+\frac{1}{b^{2}}-\frac{2 \alpha}{b}\right)} K_{(Q-2 \alpha) / b}(\kappa \ell)
\end{aligned}
$$

with

$$
\kappa^{2}=\frac{\mu}{\sin \left(\pi b^{2}\right)} .
$$

Noting that $1 / b^{2}=p+1 / 2$ and putting $\alpha_{k}=(k+2) b / 2$, one has

$$
\frac{Q-2 \alpha_{k}}{b}=p-k-\frac{1}{2}
$$

which demonstrates the consistency between the matrix model and the Liouville gravity on disk since $W_{\alpha_{k}}(\ell)$ has the same dependence on $\mu$ and $\ell$ as the one in $\left\langle O_{k}\right\rangle_{L}$ in Eq. (3.11) up to the renormalization of $L$.

\section{Conclusion and Discussion}

The result (3.6) gives an expression for the generating function of the correlation numbers of the $p$-critical one-matrix model on disk in the Liouvile frame. To confirm this proposal, we explicitly compare the one-point correlation numbers obtained from (3.6) against the FZZ one-point function Eq. (4.7) in the Boundary Liouville field theory and find the proposal consistent.

One may further evaluate the bulk $n$-point functions using (3.6). However, one cannot check their correctness at this moment, since the corresponding answers for MLG are not known yet.

Another interesting problem in both MM and MLG will be the computation of bulk-boundary correlation numbers and boundary correlation numbers on disk [21].

\section{Aknowledgements}

Authors are indebted to V. Belavin, G. Ishiki, Y. Ishimoto, M. Lashkevich, and H. Shin for useful discussions and comments. A.B. is grateful to the Center for Quantum Spacetime of Sogang University where important part of this work was performed. The research of A.B. was held within the bounds of Federal Program "Scientific and Scientific-Pedagogical personnel of innovational Russia", RFBR initiative interdisciplinary project 09-02-12446-ofi-m and RFBR-CNRS project PICS-09-02-91064. C.R. was partially supported by National Research Foundation of Korea (NRF) funded by the Korea government(MEST) with grant number 2005-0049409 and R11-2005-021. 


\section{References}

[1] A. Polyakov, "Quantum geometry of bosinic strings", Phys. Lett. B103 (1981) 207.

[2] V. Knizhnik,A. Polyakov and A. Zamolodchikov, "Fractal Structure of 2D quantum gravity", Mod. Phys. Lett. A3 (1988) 819.

[3] F. David, "Conformal Field Theories Coupled to 2-D Gravity in the Conformal Gauge", Mod. Phys. Lett. A3 (1988) 1651.

[4] J. Distler and H. Kawai, "Conformal Field Theory and 2D Quantum Gravity Or Who's Afraid of Joseph Liouville?", Nucl. Phys. B321 (1989) 509.

[5] E. Brezin and V. Kazakov, "Exactly Solvable Field Theories Of Closed Strings", Phys. Lett. B236 (1990) 144.

[6] M. Douglas and S. Shenker, "Strings in Less Than One-Dimension", Nucl. Phys. B335 (1990) 635.

[7] D. Gross and A. Migdal, "Nonperturbative Two-Dimensional Quantum Gravity", Phys. Rev. Lett. 64 (1990) 127; "A Nonperturbative Treatment Of Two-Dimensional Quantum Gravity", Nucl. Phys. B340 (1990) 333.

[8] M. Douglas, "Strings In Less Than One-Dimension And The Generalized K-D-V Hierarchies", Phys. Lett. B238 (1990) 176.

[9] V. Kazakov, "The Appearance of Matter Fields from Quantum Fluctuations of 2D Gravity", Mod. Phys. Lett A4 (1989) 2125.

[10] M. Staudacher "The Yang-Lee singularity on a dynamical planar random surface", Nucl. Phys. B336 (1990) 349.

[11] P. Di Francesco and D. Kutasov, "World sheet and space time physics in two dimensional (super) string theory", Nucl. Phys. B375 (1992) 119.

[12] P. Ginsparg and G. Moore, "Lectures on 2-D gravity and 2-D string theory (TASI 1992)", arXiv:hep-th/9304011

[13] P. Di Francesco,P. Gisparg and J. Zinn-Justin, "2-D gravity and random matrices", Phys. Rep. 254 (1995) 1.

[14] M. Goulian and M. Li, "Correlation functions in Liouville theory", Phys. Rev. Lett. 66 (1991) 2051.

[15] A. Belavin and Al. Zamolodchikov, "Moduli integrals,ground ring and four-point function in minimal Liouville gravity", Theor. Math. Phys. 147 (2006) 729; arXiv:hep-th/0510214

[16] G. Moore, N. Seiberg, M. Staudacher, "From loop to states in 2D quantum gravity", Nucl. Phys. B362 (1991) 665. 
[17] A.A. Belavin and A. Zamolodchikov, "On correlation numbers in 2D minimal gravity and matrix models", Jour. Phys. A42 (2009) 304004; arXiv: 0811.0450 [hep-th] .

[18] G. Tarnopolsky, "Five-point Correlation Numbers in One-Matrix Model", arXiv: 0912.4971 [hep-th]

[19] T. Banks, M. Douglas, N. Seiberg and S. Shenker, "Microscopic and macroscopic loops in non-perturbative two dimensional gravity", Nucl. Phys. B238 (1990) 279.

[20] V. Fateev, A. Zamolodchikov and Al. Zamolodchikov, "Boundary Liouville field theory. 1. Boundary state and boundary two point function", hep-th/0001012.

[21] K. Hosomichi, "Minimal Open Strings", JHEP 0806:029 (2008); J.-E. Bourgine and K. Hosomichi, "Boundary operators in the O(n) and RSOS matrix models", JHEP 0901:009 (2009); Jean-Emile Bourgine, Kazuo Hosomichi andIvan Kostov, "Boundary transitions of the $\mathrm{O}(\mathrm{n})$ model on a dynamical lattice", arXiv:

$0910.1581[$ hep-th] 